\title{
Minderheiten im Kontext von Globalisierung und Regionalisierung
}

Der Begriff der „Globaliserung“ ist spätestens seit den 1990er Jahren in aller Munde - auch wenn unter Historikern beispielsweise darüber gestritten wird, ob es sich hierbei tatsächlich um ein erst ab dem Zweiten Weltkrieg oder ein bereits seit Beginn der Neuzeit zu beobachtendes oder gar um ein Phänomen handelt, das so alt ist wie die Menschheit selbst. Unabhängig davon hat jedenfalls die Globalisierung im 20. und am Beginn des 21. Jahrhunderts durch die technischen, wirtschaftlichen und politischen Neuerungen speziell dieser Zeit ihr ganz spezifisches Gepräge erhalten, das in seinen Folgen vor allem in den Bereichen Wirtschaft, Politik, Kultur und Umwelt sichtbar wird.

Die Frage nach den möglichen Vor- und Nachteilen von - wie auch immer zu definierenden und empirisch zu fassenden - Prozessen der Globalisierung speziell für nationale Minderheiten und ihre Angehörigen bietet zweifellos ein eigenes umfangreiches Forschungsfeld, an dieser Stelle seien nur einige Grundgedanken dazu skizziert.

Definitionsgemäß sind nationale Minderheiten Gemeinschaften, die sich kulturell - und damit im Regelfall auch sprachlich - von der Mehrheitsbevölkerung ihres Wohnsitzstaates unterscheiden, die also kulturell anders und in diesem Anderssein vom Personenbestand her kleiner sind als die sie umgebende Bevölkerung. Wie aber ist „,kulturell“ bzw „Kultur“ zu definieren oder wenigstens inhaltlich einzugrenzen? Hierzu gibt es umfangreiche Forschungsansätze, an welchen die verschiedensten Wissenschaftsdisziplinen beteiligt sind und die je nach Perspektive und Zielrichtung der Fragestellung zu den verschiedensten Ergebnissen führen. Im Kontext der Minderheitenfrage - und womöglich nicht nur dort - dürfte es hilfreich sein, auf eine vor allem soziologisch orientierte Eingrenzung des Kulturbegriffs zurückzugreifen. Danach ist „Kultur“ das einem Kollektiv gemeinsame „Wissen“, das die im Bewusstsein der Mitglieder dieses Kollektivs verankerten Erwartungen hinsichtlich üblicher Verhaltensweisen, Werthaltungen, sozialer Deutungsmuster und Weltbilder umfasst. „Kulturen“ haben zugleich eine klare räumliche Komponente, die 
zum Begriff der „Heimat“ führt. Der Staats- und Völkerrechtler Otto Kimminich umschreibt „Heimat“

als ein Feld von Beziehungen [....], die auf den verschiedenen Ebenen des Raumes und der Zeit liegen und den Einzelmenschen sowohl mit anderen Menschen als auch mit Dingen verbinden. ${ }^{1}$

Im Begriff „Heimat“ finden sich danach objektive wie subjektive Elemente, er umfasst die drei Dimensionen Ort/Raum, Zeit und Mensch (Einzelmenschen und Gruppen) und die Beziehungen zwischen diesen Größen. ${ }^{2}$ Aus diesen Beziehungen wiederum erwächst „Kultur“ im oben umschriebenen Sinn.

Der so gefasste Kulturbegriff lässt sich auf die Mehrheits- wie auch auf die Minderheitskultur anwenden. Jedoch ergibt sich aus der - im (national-)staatlichen Maßstab - relativen Kleinheit der Minderheits- gegenüber der Mehrheitskultur ein grundsätzlicher Unterschied im Hinblick auf mögliche Auswirkungen der Globalisierung, zu welchen ua auch Migrationsbewegungen zählen. So laufen Minderheiten wesentlich schneller Gefahr, hinsichtlich ihres Personenbestandes kulturell ,,auszubluten“, also den notwendigen Mindest-Gruppenbestand vor Ort zu verlieren. ${ }^{3}$ Während also die Abwanderung eines bestimmten Quorums von Menschen des ,eigenen“ Kulturkreises die intrakulturellen Beziehungen der Mehrheit als solche in der Regel nicht wesentlich einschränkt und die Zuwanderung von Angehörigen anderer Kulturkreise im Idealfall die intrakulturellen durch interkulturelle Beziehungen ergänzt und bereichert $^{4}$, kann es für Minderheiten das kulturelle Aus bedeuten, wenn Ab- bzw Zuwanderung ein bestimmtes Maß überschreiten, so dass das ursprüngliche Beziehungsgefüge zwischen den Minderheitsangehörigen gesprengt wird. ${ }^{5}$

Allerdings ist gerade im Zuge der aktuellen Globalisierungsprozesse nicht zu übersehen, dass sich auch nationale Mehrheiten (im hier zugrundegelegten Sinne) in der Rolle von Minderheiten wiederfinden können, nämlich dann, wenn ihre relative Kleinheit auf der internationalen bzw globalen Ebene in den Fokus der Betrachtung rückt.

Vor diesem Hintergrund dürfte es noch nachvollziehbarer werden, dass - nicht nur bei Minderheiten - parallel zu den fortschreitenden Globalisierungsprozessen

\footnotetext{
${ }_{1}^{1}$ Otto Kimminich [1989]: Das Recht auf die Heimat, Bonn, S 42.

2 O. Kimminich aaO, S 48 .

${ }^{3}$ Die von der Europäischen Kommission in Auftrag gegebene Studie „Euromosaic“ bestätigt zB einen direkten Zusammenhang zwischen der (hier absoluten) Größe - im Sinne von Sprecherzahl - einer Sprache und ihrer Überlebensfähigkeit, wobei sie die kritische Grenze vorsichtig bei 300.000 Sprechern ansetzt. Eine weitere gefährliche Schwelle ist danach bei Sprachgemeinschaften mit weniger als 50.000 Angehörigen überschritten, vgl dazu Europäische Kommission (Hrsg) [1996]: Euromosaic. Produktion und Reproduktion der Minderheitensprachgemeinschaften in der Europäischen Union, Luxemburg.

${ }^{4}$ Vgl dazu historisch Matthias Theodor Vogt [2009]: DE FAVORE PEREGRINORUM. Minderheiten- und Fremdenfreundlichkeit am Wiederbeginn europäischer Staatlichkeit, in: EJM 4-2009, S 164-176.

5 Zur Problematik der möglichen Kollision nationalstaatlicher Minderheitenschutzvorkehrungen mit den EU-Binnenmarktfreiheiten und neuen Lösungsperspektiven infolge des Vertrags von Lissabon vgl Waldemar Hummer [2011]: Minderheitenschutz im Recht der EU vor und nach dem Inkrafttreten des Vertrages von Lissabon - vom bloßen Diskriminierungsverbot zu ,affirmative actions“, in: EJM 2-2011, S 81-102.
} 
immer stärkere Tendenzen zur Regionalisierung zu beobachten sind ${ }^{6}$ - erinnert sei hier nochmals an die Rolle speziell der räumlichen Dimension bei der Herausbildung von Kulturen. Der Begriff „Regionalismus“ bezeichnet sowohl

- die Identifikation von Menschen mit einer Region als auch

- das Streben nach Schaffung regionaler Gebietskörperschaften oder Institutionen, die (zB durch eigene Verwaltungs- und idealerweise auch Gesetzgebungszuständigkeiten) zu autonomen Entscheidungen befugt sind oder das Streben nach Ausweitung der Kompetenzen solcher Körperschaften oder Institutionen.

Auf (inner-)staatlicher Ebene geht es also um regionale Selbstverwaltung bzw regionale Demokratie oder zumindest um Dezentralisierung (,devolution“"), wobei „Regionen" Gebiete sind, die kleiner sind als die betreffenden Staaten, aber jedenfalls größer als Städte oder Gemeinden. Hinzu kommen aber auch grenzüberschreitende Regionen wie die sog Europaregionen und schließlich können aus globaler Perspektive auch Gebiete, die kleiner sind als die Welt, aber größer als Staaten, so zB die EU, der Verband Südostasiatischer Nationen ASEAN oder die Staaten des Nordamerikanischen Freihandelsabkommens NAFTA, als Regionen bezeichnet werden. Angesichts des hier eingegrenzten Kulturbegriffs ist es auf jeder dieser Ebenen sinnvoll, wichtig oder gar entscheidend, bei der Ziehung von Regionengrenzen historischkulturelle Gegebenheiten, zB die Existenz und Siedlungsstruktur historisch gewachsener Minderheiten, mit zu berücksichtigen.

Im Ergebnis zeichnen sich hier - auch, aber nicht nur aus der Minderheitenperspektive - Entwicklungen ab, die neben Risiken (zB Sicherheitsrisiken durch Machtkämpfe) auch Chancen bieten. Die Chancen, die sich speziell aus der regionalen Selbstverwaltung/Demokratie für (va geschlossen siedelnde) Minderheiten ergeben, wurden im EJM bereits zur Sprache gebracht. ${ }^{7}$ Erinnert sei an dieser Stelle vor allem auch an die Chancen auf wirtschaftlichen Mehrwert für alle Beteiligten, die sich mehrsprachigen Regionen mit autonomen Kompetenzen eröffnen. ${ }^{8}$ Ganz allgemein könnte die Regionalisierung im Zusammenspiel mit der Globalisierung und unter Nutzung der speziell auf der regionalen Ebene bestehenden Möglichkeiten der grenzüberschreitenden intrakulturellen wie interkulturellen Kohäsion ${ }^{9}$ schließlich zu einer Art Gleichgewicht ,zentrifugaler“" und ,zentripetaler“ Kräfte führen.

\footnotetext{
${ }^{6} \mathrm{Zu}$ neueren Entwicklungen auf der europäischen Ebene vgl Beate Sibylle Pfeil [2008]: Neue Empfehlung beim Europarat zur Stärkung des Regionalismus, in: EJM 1-2008, S 65-71; B. S. Pfeil [2010]: Regionale Selbstverwaltung beim Europarat: Der neue „Bezugsrahmen für regionale Demokratie“, in: EJM 2-2010, S 114-128.

${ }^{7}$ Allgemein dazu Christoph Pan [2008]: Die Bedeutung von Minderheiten- und Sprachschutz für die kulturelle Vielfalt Europas, in: EJM 1-2008, S 25.

8 Vgl B. S. Pfeil [2010]: Neue Perspektive beim Europarat: Minderheitensprachen als Instrument der Regionalentwicklung, in: EJM 2-2010, S 100-113; Vladimir Kreck [2011]: Zur Diskussion gestellt: Autochthone Minderheiten und ökonomischer Mehrwert, in: EJM 3-2011, S 149-160; allgemein zum Thema „Mehrwert durch Minderheiten“ M. T. Vogt [2009]: Mehrwert durch Minderheiten? Das VIII. Collegium PonTEs Görlitz-Zgorzelec-Zhořelec, in: EJM 2-2009, S 55-71.

9 Vgl dazu allgemein B. S. Pfeil [2011]: (Inter-)Kulturelle Kohäsion in grenzübergreifenden Kultur- und Sprachräumen. Vorwort der Redaktion, in: EJM 4-2011, S211-214. Zu neuen Möglichkeiten grenzüberschreitender Zusammenarbeit im Rahmen der EU vgl Walter Obwexer/Esther Happacher [2010]: Rechtsfragen der Gründung eines Europäischen Verbundes für territoriale Zusammenarbeit (EVTZ) am Beispiel
} 
Hier kommt das durch die Wortneuschöpfung „Glokalisierung“ - als Zusammensetzung der Begriffe „Globalisierung“ und „Lokalisierung“ - umschriebene Phänomen ins Spiel. ${ }^{10}$ Nach Peter J. Weber bezeichnet „Glokalisierung“ die

[...] konstitutive Komplementarität von gleichzeitigen, gekoppelten und dabei auch gegenläufigen Prozessen der Globalisierung und der Lokalisierung [...],

Globalisierung ist danach also „mit Regionalisierung und Lokalisierung verbunden“. Im Idealfall führt diese Kombination zu einer aus der Sicherheit der eigenen regionalkulturellen Identität erwachsenden und durch die Globalisierung begünstigten Weltoffenheit, in deren Rahmen auch die anderen Kulturen geachtet und respektiert werden.

Peter J. Weber beleuchtet in seinem Beitrag die Auswirkungen von Glokalisierungsprozessen speziell auf den Gebrauch und die Bedeutung von Regional- und Minderheitensprachen, wobei auch und gerade hier deren (natürlicher) Mehrwert zur Sprache kommt.

Speziell unter dem Gesichtspunkt „Regionalismus“ im Rahmen der Glokalisierung bieten auch das Elsass und der im EJM schon mehrfach behandelte Fall Cornwall ${ }^{11}$ interessante Beispiele.

Die Geschichte des Elsass ist komplex und geprägt durch dessen geographische Lage an der Schnittstelle zweier großer europäischer Sprach- und Kulturräume. Hier kommt ein zusätzlicher Aspekt des Regionalismus zum Tragen: Die speziell in historisch gemischtsprachigen Gebieten häufiger zu beobachtende Rückbesinnung fast ausschließlich auf die regionale Identität, die auch als Ausweg aus dem Dilemma der politischen Vereinnahmung betrachtet wird - wobei sich solche Vereinnahmungstendenzen historisch gesehen seit dem nationalstaatlichen Zeitalter deutlich verstärkt haben. Die aktuellen Regionalisierungsbestrebungen im Elsass variieren zwar hinsichtlich Intensität und der Bandbreite ihrer politischen Zielsetzungen, bei allen politischen Gruppierungen geht es aber letztlich (auch) um die Rettung der spezifisch „elsässischen“, mehrsprachigen Identität, die nicht nur die Standardsprachen Französisch und Deutsch, sondern auch die im Elsass gesprochenen alemannischen Dialekte umfasst.

Ein leidenschaftlicher Verfechter nicht nur eines elsässischen Regionalismus in diesem Sinne, sondern auch einer europäischen Einigung auf der Grundlage eines Bündnisses zwischen Frankreich und Deutschland - unter Nutzung des elsässischen Brücken- und Vermittlerpotentials - war der aus einem gemischtsprachigen Elternhaus stammende elsässische Schriftsteller René Schickele (1883-1940). Áine McGillicuddy beschreibt in ihrem Beitrag das Leben Schickeles als Unverstandener ,zwischen den Stühlen" und erläutert insbesondere dessen eindrucksvolles politisches Vermächtnis. Mit seinen Visionen eines geeinten Europas bei gleichzeitiger Achtung

der Europaregion Tirol, in: EJM 2-2010, S 75-99; Alice Engl/Andreas Eisendle [2011]: Der Europäische Verbund für territoriale Zusammenarbeit (EVTZ) als Kooperationsinstrument in pluri-ethnischen Grenzregionen, in: EJM 4-2011, S215-238.

${ }^{10}$ Der Begriff „Glokalisierung“ ist erstmals in den 1980er Jahren aufgekommen.

11 Vgl John Fleet [2008]: Cornish identity at risk - a rundown on the present situation in Cornwall, in: EJM 4-2008, S 237-240; J. Fleet [2008]: The work of the Cornish organisation CERES, in: EJM 3-2008, S 195-198. Vgl auch die Hinweise in Fußnote 12. 
regionaler Identitäten erweist sich Schickele zugleich als Vordenker der Glokalisierung auf europäischer Ebene.

Wie im Elsass, so gibt es auch in Cornwall nach einer langen Phase der Assimilierung mittlerweile starke Regionalisierungsbestrebungen, die mit dem Wunsch nach einer Wiederbelebung der regionalen (keltischen) Sprache und Kultur verbunden sind. Dies umso mehr, als den anderen keltisch geprägten Regionen Großbritanniens - Schottland, Wales und Nordirland - im Zuge eines Dezentralisierungsprozesses (,,devolution“) bereits seit 1998 gewisse Teilautonomien eingeräumt wurden. Überdies wurden die Schotten, Iren und Waliser dem Schutzbereich des 1998 ratifizierten Europarat-Rahmenübereinkommens zum Schutz nationaler Minderheiten unterstellt - nicht aber die Kornen. ${ }^{12}$ Der Beitrag Jim Pengellys zu causa Cornwall liefert nicht nur weitere $\mathrm{zB}$ historische Hintergrundinformationen $\mathrm{zu}$ den kornischen Aspirationen, sondern lässt zugleich erkennen, mit welcher Vehemenz die entsprechende Diskussion zum Teil geführt wird.

Auf eine Minderheitenproblematik ganz anderer Art verweist Ronnie Moore in seinem Beitrag über die Fahrenden in Irland (Irish Travellers). Die Fahrenden genießen in Irland einen rechtlichen Sonderstatus und sind als eigene zu schützende Gemeinschaft anerkannt. Anhand der Fahrenden zeigt sich, dass die oben zugrundegelegte Eingrenzung des Begriffs „Kultur“ auf den (Sonder-)Fall der Fahrenden - wie überhaupt auf alle jedenfalls ursprünglich ,fahrenden“ Gemeinschaften - nur bedingt anwendbar ist. Zwar findet man auch hier die Dimensionen „Zeit“ und „Mensch (Einzelmenschen und Gruppen)“ und die Beziehungen zwischen diesen Größen, jedoch erstreckt sich hier die Dimension „Raum“ jedenfalls nicht auf ein ganz bestimmtes Gebiet, sondern ist durch das Streben nach regelmäßigem Ortswechsel charakterisiert. Auch wenn ein Großteil sowohl der Travellers als auch der Angehörigen anderer traditionell fahrender Gemeinschaften in Europa längst sesshaft geworden ist, halten die meisten, so Moore in Bezug auf die Irish Travellers, am „,erklärten Willen, nach Belieben zu reisen“ als Grundlage ihrer „,kulturellen Unverwechselbarkeit" fest. Genau in diesem Punkt unterscheiden sich die fahrenden Gemeinschaften also kulturell grundsätzlich vom „Rest der Welt“, einer Welt, deren politische und staatsrechtliche Systeme sich trotz Globalisierung am Prinzip der Sesshaftigkeit (welches zB die Bildung von Privateigentum einschließt) orientieren. Genau hier beginnt wohl auch die Tragik dieser Gemeinschaften, die schon aufgrund ihres in dieser Hinsicht wesentlichen Andersseins einem enormen Assimilierungsdruck und einem besonders hohen Diskriminierungsrisiko ausgesetzt sind, in dessen Gefolge althergebrachte soziale Beziehungsgefüge innerhalb dieser Gruppen zu zerbrechen drohen. Ronnie Moore konzentriert sich in seinem Beitrag vor allem auf die dramatischen sozialen und gesundheitlichen Auswirkungen dieser Entwicklungen und verweist damit zugleich auf einen wichtigen Teilaspekt der ver-

\footnotetext{
12 Vgl dazu Ian Saltern [2011]: The Cornish National Minority Report 2 (Excerpts). Including the Cornish - a unique case for recognition, in: EJM 3-2011, S 187-205; B. S. Pfeil [2011]: Die Kornen - eine Minderheit im Sinne des Rahmenübereinkommens zum Schutz nationaler Minderheiten? Eine Einführung zum „Cornish National Minority Report 2“, in: EJM 3-2011, S 181-186.
} 
einfacht „Romafrage“ genannten Problematik in Europa ingesamt. ${ }^{13}$ Hier sind nach wie vor besonders durchdachte und sensible Lösungskonzepte gefragt, und zwar auf der politischen wie auf der kulturellen und sozialen Ebene, auf der nationalen wie auf der internationalen bzw ,globalen“ Ebene. Ob und inwieweit die Globalisierung selbst, die jedenfalls die in neuerer Zeit ergriffenen konzertierten politischen Maßnahmen auf internationaler und europäischer Ebene ${ }^{14}$ erleichtert und außerdem in vieler Hinsicht auf Mobilität setzt, hier Lösungsansätze zu liefern vermag, bleibt abzuwarten.

Beate Sibylle Pfeil

13 Vgl dazu allgemein Helmut Tichy [2011]: Roma - Wohin geht die Reise? Roma-Themen in internationalen Organisationen, in: EJM 1-2011, S 49-63; Rudolf Sarközi [2011]: Rom sein in Österreich, in: EJM 1-2011, S65-69.

$14 \mathrm{Vgl}$ dazu H. Tichy aa O. 